\title{
Scalable Structured Prediction for Richly Structured Socio-Behavioral Data
}

\author{
Keynote \\ Lise Getoor \\ University of California, Santa Cruz \\ USA \\ getoor@soe.ucsc.edu
}

\begin{abstract}
Online recommender systems, content-provider sites, and social media platforms provide richly structured socio-behavioral data. However, using this noisy and incomplete data to make decisions and recommendations is challenging. It often requires complex forms of structured prediction that rely on both the logical structure in the domain and probabilistic dependencies among interlinked entities. In this talk, I will describe some common inference patterns that are useful for socio-behavioral networks and introduce probabilistic soft logic (PSL). PSL is a highly scalable open-source probabilistic programming language being developed within my group that is well-suited for structured prediction over socio-behavioral data. Finally, I will review some of our recent work using PSL for hybrid recommender systems, explanation, and fair decision making.

\section{ACM Reference Format:}

Lise Getoor. 2018. Scalable Structured Prediction for Richly Structured SocioBehavioral Data: Keynote. In Twelfth ACM Conference on Recommender Systems (RecSys '18), October 2-7, 2018, Vancouver, BC, Canada. ACM, New York, NY, USA, 1 page. https://doi.org/10.1145/3240323.3267109
\end{abstract}

\section{BIO}

Lise Getoor is a professor in the Computer Science Department at the University of California, Santa Cruz. Her research areas include machine learning, data integration and reasoning under uncertainty, with an emphasis on graph and network data. She has over 200 publications and extensive experience with machine learning and probabilistic modeling methods for graph and network data. She is a Fellow of the Association for Artificial Intelligence, an elected board member of the International Machine Learning Society, served on the board of the Computing Research Association (CRA), and was co-chair for ICML 2011. She is a recipient of an NSF Career Award and twelve best paper and best student paper awards. She received her PhD from Stanford University in 2001, her MS from UC Berkeley, and her BS from UC Santa Barbara, and was a professor in the Computer Science Department at the University of Maryland, College Park from 2001-2013.

Permission to make digital or hard copies of part or all of this work for personal or classroom use is granted without fee provided that copies are not made or distributed for profit or commercial advantage and that copies bear this notice and the full citation on the first page. Copyrights for third-party components of this work must be honored.

For all other uses, contact the owner/author(s).

RecSys '18, October 2-7, 2018, Vancouver, BC, Canada

(c) 2018 Copyright held by the owner/author(s).

ACM ISBN 978-1-4503-5901-6/18/10.

https://doi.org/10.1145/3240323.3267109 